\title{
Subcycle Controlled Charge-Directed Reactivity with Few-Cycle Midinfrared Pulses
}

\author{
I. Znakovskaya, ${ }^{1}$ P. von den Hoff, ${ }^{2}$ G. Marcus, ${ }^{1,3}$ S. Zherebtsov, ${ }^{1}$ B. Bergues, ${ }^{1}$ X. Gu, ${ }^{1}$ Y. Deng, ${ }^{1}$ M. J. J. Vrakking, ${ }^{4}$ \\ R. Kienberger, ${ }^{1}$ F. Krausz, ${ }^{1}$ R. de Vivie-Riedle, ${ }^{2}$ and M. F. Kling ${ }^{1,5}$ \\ ${ }^{1}$ Max-Planck Institute of Quantum Optics, Hans-Kopfermann-Strasse 1, D-85748 Garching, Germany \\ ${ }^{2}$ Department für Chemie und Biochemie, Ludwig-Maximilians-Universität München, Butenandt-Strasse 11, \\ D-81377 München, Germany \\ ${ }^{3}$ Department of Applied Physics, The Benin School of Engineering and Computer Science, \\ The Hebrew University of Jerusalem, Jerusalem, 91904, Israel \\ ${ }^{4}$ Max-Born-Institut, Max-Born Strasse 2A, D-12489 Berlin, Germany \\ ${ }^{5}$ J. R. Macdonald Laboratory, Kansas State University, Cardwell Hall 116, Manhattan, Kansas 66506, USA
}

(Received 19 August 2011; published 8 February 2012)

\begin{abstract}
The steering of electron motion in molecules is accessible with waveform-controlled few-cycle laser light and may control the outcome of light-induced chemical reactions. An optical cycle of light, however, is much shorter than the duration of the fastest dissociation reactions, severely limiting the degree of control that can be achieved. To overcome this limitation, we extended the control metrology to the midinfrared studying the prototypical dissociative ionization of $\mathrm{D}_{2}$ at $2.1 \mu \mathrm{m}$. Pronounced subcycle control of the directional $\mathrm{D}^{+}$ion emission from the fragmentation of $\mathrm{D}_{2}^{+}$is observed, demonstrating unprecedented charge-directed reactivity. Two reaction pathways, showing directional ion emission, could be observed and controlled simultaneously for the first time. Quantum-dynamical calculations elucidate the dissociation channels, their observed phase relation, and the control mechanisms.
\end{abstract}

DOI: 10.1103/PhysRevLett.108.063002

PACS numbers: 33.80.Wz, 31.15.A-, 31.70.Hq

Coherent control of photochemical reactions has been achieved in the past decades by manipulating the laser frequency, phase, and polarization [1]. An exciting novel perspective is to directly control a molecular rearrangement by the waveform of a carrier-envelope phase (CEP) stabilized few-cycle laser pulse $E(t)=E_{0}(t) \cos (\omega t+\varphi)$ for an envelope function $E_{0}(t)$, angular carrier frequency $\omega$, and $\operatorname{CEP} \varphi$. The CEP adds a new dimension to the existing control parameters and offers the potential to very significantly enhance the possibilities for achieving control over chemical reactions by steering the electrons [2]. Note that the CEP control is related to but not identical to an $\omega-2 \omega$ phase control (see, e.g., [3,4]). The steering of electron motion in molecules can be achieved by superimposing two or more electronic states of different parity with a well-defined phase relationship that depends on the CEP. The CEP guided electron motion inside the molecule may break or even form chemical bonds in the sense of charge-directed reactivity [5].

CEP-stabilized few-cycle pulses in the near infrared have been used to control the dissociative ionization of diatomic molecules [6-11]. To facilitate an efficient CEP control, it is necessary to adjust the time scale of the fewcycle pulse to the time scale of the nuclear motion. As an example, the dissociation time for the molecular hydrogen ion via bond softening (BS) [12] may be estimated as approximately half of a vibrational period, corresponding to $12 \mathrm{fs}$ [13].

The dependence of the CEP-controlled electron localization in molecular hydrogen has been explored as a function of the laser intensity [14] and pulse duration [15]. Previous experiments and theory have indicated that the CEP control decreases exponentially with the number of laser cycles [6,15-17], making it necessary to keep the number of laser cycles for CEP control small. Therefore, the application of this control scheme to larger molecules with longer dissociation times necessarily requires larger wavelengths $\lambda$ with optical periods $T=\lambda / c$ (where $c$ is the speed of light) that are significantly longer in time than their counterparts in the near infrared. Here, we open the path to CEP control at larger wavelengths via experimentally and theoretically exploring the subcycle control of the dissociative ionization of $\mathrm{D}_{2}$ in intense few-cycle CEP-stable midinfrared laser fields. The demonstration of efficient control of charge-directed reactivity in this proof-of-principle study is relevant to the control of larger molecules and complex molecular processes. As an example, the branching ratio at conical intersections is expected to be strongly dependent on charge localization induced by a midinfrared few-cycle pulse [18].

Midinfrared pulses at $2.1 \mu \mathrm{m}$ were generated at a repetition rate of $1 \mathrm{kHz}$ by an optical parametric chirped-pulse amplification system [19]. The experimental setup essentially resembles the one described in [20]. The experiments reported here were carried out with 25 fs linearly polarized laser pulses. The relative CEP $\varphi_{\text {CEP }}$ in the experiment was varied by changing the amount of dispersive material in the beam path. The laser pulses were focused to an intensity of $(6.2 \pm 1.5) \times 10^{13} \mathrm{~W} \mathrm{~cm}^{-2}$ in the center of a velocity-map imaging spectrometer [21] with a $\mathrm{CaF}_{2}$ lens $(f=30 \mathrm{~cm})$, where they intersected an effusive $\mathrm{D}_{2}$ gas jet. 


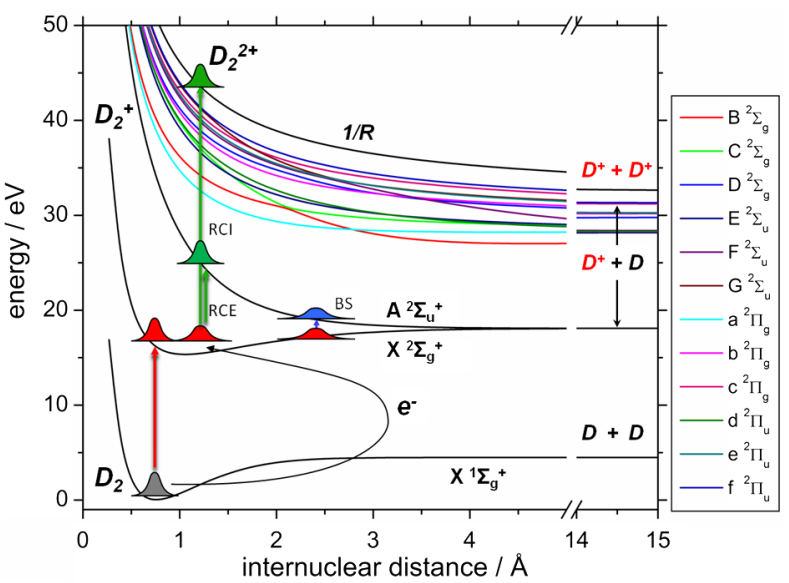

FIG. 1 (color online). Relevant potential energy surfaces of $\mathrm{D}_{2}, \mathrm{D}_{2}^{+}$, and $\mathrm{D}_{2}^{2+}$ obtained by ab initio calculations described in the text. The higher lying excited electronic states of $\mathrm{D}_{2}^{+}$above the $A$ state are labeled alphabetically accordingly to their symmetry. The red arrow indicates tunnel ionization of $\mathrm{D}_{2}$, the blue arrow bond softening (BS), and the green arrows recollision induced excitation (RCE) and ionization (RCI).

The principle pathways for the dissociative ionization of $\mathrm{D}_{2}$ are displayed in Fig. $1 . \mathrm{D}_{2}^{+}$is produced from $\mathrm{D}_{2}$ by tunnel ionization in the intense laser field (red arrow). Thereby a nuclear wave packet is launched on the $X^{2} \Sigma_{g}^{+}$ potential. This initial step can be followed by several processes leading to the dissociation of the molecular ion: (i) bond softening via the laser-induced coupling of the $X^{2} \Sigma_{g}^{+}$and $A^{2} \Sigma_{u}^{+}$states [12] (BS, blue arrow), (ii) recollisional excitation (RCE), (iii) recollisional ionization (RCI) (green arrows), and (iv) laser-induced excitation to higher lying $\mathrm{D}_{2}^{+}$states (not shown). In our study, two pathways [(i) and (ii)] leading to the directional $\mathrm{D}^{+}$ion emission can be differentiated and controlled within the same experiment. The results, including the complex phase relation between the different channels, can be understood from our time-dependent Schrödinger equation calculations.

Figure 2(a) shows a cut for $p_{z}=0$ through the 3D momentum distribution of the $\mathrm{D}^{+}$ions. The electric field was polarized along the $p_{y}$ axis. The corresponding measured $\mathrm{D}^{+}$kinetic energy spectrum, integrated over the full solid angle, is displayed in Fig. 2(b) (black solid line). The spectrum reveals 4 regions, which are separated in Fig. 2(a) by dashed circles. The most intense contribution in the spectrum between 0 to $1 \mathrm{eV}$ is assigned to BS. Three additional contributions can be identified at higher energies in the ranges 1-4 eV, 4-9 eV, and 9-13 eV. The identification of these channels together with our theoretical treatment is discussed below.

The directional $\mathrm{D}^{+}$ion emission as a function of the measured phase $\varphi_{\text {CEP }}$ (which exhibits a phase offset with respect to the absolute $\operatorname{CEP} \varphi$ ) and momentum $p$ is analyzed by the angle-integrated asymmetry parameter
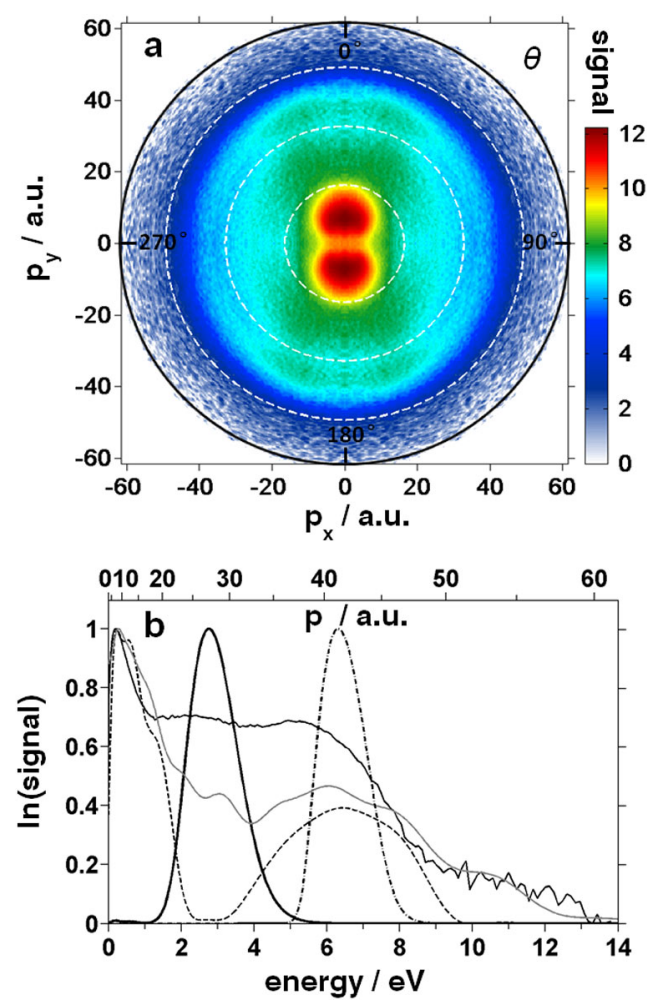

FIG. 2 (color online). (a) Inverted, CEP-averaged twodimensional $\mathrm{D}^{+}$momentum distribution $\left(p_{y}\right.$ versus $p_{x}$ at $p_{z}=$ $0)$. The laser is polarized along the $p_{y}$ axis. The momentum distribution was left-right as well as up-down symmetrized to increase the signal-to-noise ratio. The dashed circles separate the four contributions discussed in the text. A logarithmic color scale was used. (b) Angular integrated kinetic energy spectrum of $\mathrm{D}^{+}$ions obtained from the experimental data (black solid line) and calculated spectra for the bond-softening dissociation involving only the $X$ and $A$ states of $\mathrm{D}_{2}^{+}$(dashed line) and also the 11 higher states of $\mathrm{D}_{2}^{+}$shown in Fig. 1 (gray solid line). The theoretical results for the dissociation via recollision induced excitation and ionization are shown as bold and dash-dotted black lines, respectively.

$$
A\left(p, \varphi_{\mathrm{CEP}}\right)=\frac{N_{\text {up }}\left(p, \varphi_{\mathrm{CEP}}\right)-N_{\mathrm{down}}\left(p, \varphi_{\mathrm{CEP}}\right)}{N_{\mathrm{up}}\left(p, \varphi_{\mathrm{CEP}}\right)+N_{\mathrm{down}}\left(p, \varphi_{\mathrm{CEP}}\right)}
$$

with the ion yields $N_{\text {up }}\left(p, \varphi_{\text {CEP }}\right)$ and $N_{\text {down }}\left(p, \varphi_{\text {CEP }}\right)$ in the up and down directions, respectively. The ion yields were integrated over an opening angle of $20^{\circ}$ along the laser polarization axis. The asymmetry $A(p, \varphi)$ is shown in Fig. 3(a), where the experimental phase offset was calibrated to achieve best agreement between theory and experiment in the CEP-dependent asymmetry oscillation for the BS channel. A high degree of asymmetry (with an amplitude exceeding 0.2) is found at energies below $1 \mathrm{eV}$, the energy range corresponding to the BS channel. The asymmetry in this channel shows a tilt in kinetic energy of its oscillation with CEP and an additional phase jump at $0.17 \mathrm{eV}$, which has not been observed in an earlier study in the near infrared [7]. A second asymmetry 


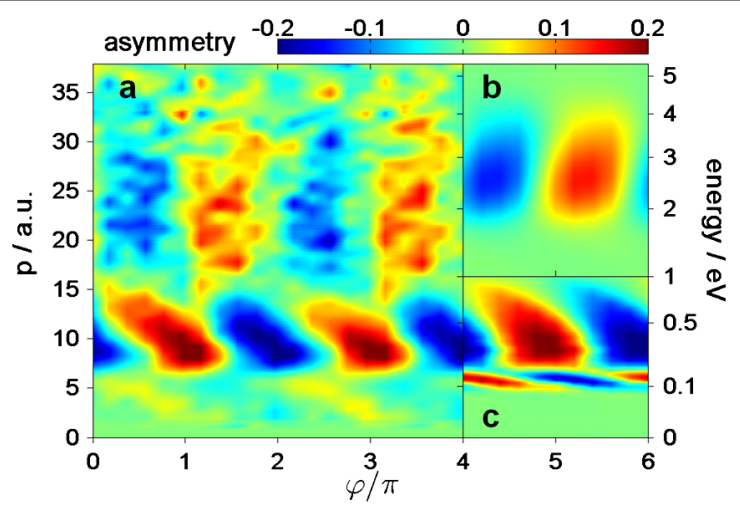

FIG. 3 (color online). (a) $\mathrm{D}^{+}$ion emission asymmetry as a function of the fragment momentum (corresponding kinetic energy scale on the right) and relative phase $\varphi_{\mathrm{CEP}}$ of the $\mathrm{D}^{+}$ ion emission obtained by integrating over a full opening angle of $20^{\circ}$ along the laser polarization axis. (b) Asymmetry obtained by the calculations for the RCE channel. (c) Same as (b) for the BS channel.

contribution in the energy range $1-4 \mathrm{eV}$ has a weaker (maximum 0.1) amplitude and its oscillation with CEP is independent of kinetic energy. A $\pi$ phase jump between the two channels is observed at approximately $1 \mathrm{eV}$. The observation of CEP control for various dissociation channels and their phase relation is very remarkable when compared to earlier results obtained in the near infrared $[6,7]$, where this was not feasible so far. Above $4 \mathrm{eV}$ only vanishingly small or no asymmetry was measured under the present experimental conditions (compared to asymmetry contributions up to approximately $8 \mathrm{eV}$ in the near infrared [6]).

We model the dissociative ionization of $\mathrm{D}_{2}$ using our previously published approach to treat the coupled electron and nuclear wave packet dynamics [17]. For details, see the Supplemental Material [22]. The required potential energy surfaces were calculated using the quantum chemistry package MOLPRO [23] on the CASSCF $(1,15)$ level of theory using the $(11 s, 7 p) \rightarrow[6 s, 5 p]$ contracted Gaussian basis set introduced by Whitten and Huzinaga [24,25]. The calculations were performed for molecules, aligned along the laser polarization axis, under the experimental laser conditions.

To simulate the BS mechanism we projected the vibrational ground state wave function of the $\mathrm{D}_{2}$ molecule on the $X^{2} \Sigma_{g}^{+}$potential and assumed that ionization only takes place at the peaks of the electric field (corresponding to the highest ionization probability). In the case of the RCE mechanism the wave packet is initially propagated on the $X^{2} \Sigma_{g}^{+}$surface and projected to the $A^{2} \Sigma_{u}^{+}$surface (for RCE) and the $\mathrm{D}_{2}^{2+}$ surface (for RCI) at the time of recollision (which is $\frac{2}{3}$ of an optical cycle, i.e., $4.7 \mathrm{fs}$ at $2.1 \mu \mathrm{m}$ [26]).

Figure 2(b) shows the theoretical results for different dissociation channels and conditions. The dashed curve shows the calculated $D^{+}$kinetic energy spectrum for the laser-induced dissociation mechanism including only the $X^{2} \Sigma_{g}^{+}$and $A^{2} \Sigma_{u}^{+}$surfaces of $\mathrm{D}_{2}^{+}$. The gray curve shows the result, where also the 11 higher excited states shown in Fig. 1 have been included. The experimental spectrum below $2 \mathrm{eV}$ is reproduced well by both theoretical results and assigned to the BS channel. The contribution in the energy range 4-9 $\mathrm{eV}$ results from the instantaneous population of the $A$ state within the ionizing half-cycle. The high-energy tail of the measured $\mathrm{D}^{+}$spectrum above $9 \mathrm{eV}$ is only captured by the calculations that include the higher excited states [gray curve in Fig. 2(b)]. Such a high-energy tail has also been observed in earlier experimental work at $760 \mathrm{~nm}$ [6], but has not been explained yet. The spectrum resulting from a calculation for the RCE dissociation is shown as a bold black curve. The resulting contribution can explain the strong experimental signal between 2 and $4 \mathrm{eV}$. We also computed the spectrum resulting from RCI (dash-dotted curve), which may occur due to the high ponderomotive potential in the midinfrared and thus high recollision energy (reaching up to $84.5 \mathrm{eV}$ at $6.2 \times$ $10^{13} \mathrm{~W} \mathrm{~cm}^{-2}$ ). Double ionization results in the Coulomb explosion of the molecule and production of two $\mathrm{D}^{+}$fragments that are emitted into opposite directions along the laser polarization axis. $\mathrm{D}^{+}$fragments from this dissociation channel cannot produce any asymmetry in the ion emission, and spectral overlap with other channels would reduce or even mask observable asymmetries.

The calculated asymmetries corresponding to the dissociation of $\mathrm{D}_{2}^{+}$by $\mathrm{RCE}$ and $\mathrm{BS}$ are shown in Figs. 3(b) and 3(c), respectively (each channel is calculated separately). In order to facilitate a comparison of the theoretical results with the experimental data, the asymmetries were calculated assuming ionization by the 5 most intense peaks of the laser electric field. Inclusion of further peaks did not lead to significant changes. Although volume averaging effects have not been considered in our theoretical treatment, the asymmetry amplitude and its energydependent oscillatory behavior with the CEP are well reproduced by the calculations. Note that the two calculations were performed for the same CEP $\varphi$ and were not shifted against each other afterwards. The phase jump at $0.17 \mathrm{eV}$ in the BS channel is also reproduced.

In the calculations shown in Fig. 3(b) we included only the first recollision after the initial ionization. Calculations, considering later recollisions, are contained in the Supplemental Material [22] and indicate that the experimentally observed lower kinetic energies in the RCE channel (below $2 \mathrm{eV}$ ) may arise from subsequent recollisions. We find that at $2.1 \mu \mathrm{m}$ the first three recollision events result in the same sign for the asymmetry. Autoionization and/or photoionization of highly excited neutral $\mathrm{D}_{2}$ molecules, which is more likely in the tunneling regime [27], may also produce an asymmetry in the breakup of hydrogen [28]. These mechanisms may explain some remaining differences between the experimental and theoretical data. 
Their theoretical treatment at long wavelengths and high laser intensities is, however, beyond current computing capabilities and was therefore not considered here.

Within the framework of our model, the subcycle control is understood as follows: the laser-induced coupling of multiple electronic states produces a coherent superposition of these states, which results in the localization of the electron in $\mathrm{D}_{2}^{+}$and the asymmetric breakup of the molecule. The phase of the coherent superposition, defining the localization of the electron, is controlled by the phase of the applied electric field (here via the CEP). In the case of the BS mechanism an initially prepared wave packet on the $X$ state of $\mathrm{D}_{2}^{+}$moves toward the outer turning point of the potential and is efficiently coupled to the $A^{2} \Sigma_{u}^{+}$state at large internuclear distances (see Fig. 1). Our quantum mechanical calculations reproduce the observed tilt of the asymmetry with kinetic energy and the phase jump at $0.17 \mathrm{eV}$ (see Fig. 3). The phase jump likely originates from the laser coupling with the slowest components of the dissociating wave packet. Future studies involving semiclassical approaches [29] and full two-electron calculations including correlation effects [30] might be helpful to elucidate this aspect in more detail.

The higher energy contribution in the BS channel between 4 and $10 \mathrm{eV}$ (see Fig. 2) originates from the direct, laser-induced population of the $A$ state right after ionization with subsequent dissociation. As this fast component of the wave packet is not coupled back to the $X$ state, the symmetry is not broken and thus no asymmetry is observable in this energy range. In the RCE mechanism the $A$ state is populated by recollision at later times. Because of the potential shape, this results in lower kinetic energies in the range between 2 and $4 \mathrm{eV}$. During the dissociation the remaining laser couples population from the $A$ state back to the $X$ state, leading to a coherent superposition of both states and an observable asymmetry. The electronic states are superimposed in the BS and RCE mechanisms with different phases, resulting in the $\pi$ phase jump in the asymmetry at $1 \mathrm{eV}$.

The wavelength dependence of the asymmetry is further analyzed by calculations shown in Fig. 4. The energy integrated asymmetry amplitudes for the BS channel considering ionization at the peak electric field (for $\varphi=0$ ) using a 3.5-cycle pulse for different wavelengths are shown in Fig. 4(a). The asymmetry oscillates close to zero around $800 \mathrm{~nm}$, explaining the difficulty to observe the CEP control in this channel with near-infrared few-cycle pulses. With increasing wavelength, the asymmetry amplitude reaches higher values. This behavior supports our initial statement that the duration of the few-cycle laser pulse has to match the time scale of the chemical reaction. A further example for 2-cycle pulses is shown in the Supplemental Material [22]. The wavelength dependence of the RCE dissociation is shown in Fig. 4(b) as a function of the $\mathrm{D}^{+}$ fragment kinetic energy and the recollision time. With $\lambda / \mu \mathrm{m}$

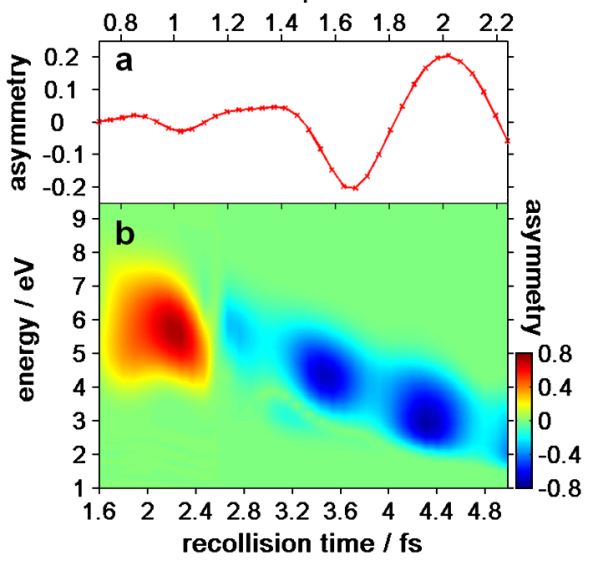

FIG. 4 (color online). (a) Calculated energy integrated asymmetry amplitude in the BS channel for various laser wavelength assuming a 3.5-cycle pulse. (b) Calculated asymmetry map for the RCE channel as a function of kinetic energy and recollision time (corresponding to the wavelength given on the top axis).

increasing wavelength, the recollision time increases, leading to an excitation to the $A$ state at larger internuclear distances. As a result the kinetic energy of the $\mathrm{D}^{+}$ fragments is decreasing from initially around $6 \mathrm{eV}$ at $760 \mathrm{~nm}$ to less than $3 \mathrm{eV}$ at $2.1 \mu \mathrm{m}$.

The sign of the asymmetry in the BS and RCE dissociation channels is strongly dependent on the wavelength. Therefore, the relative phase between the CEP-dependent asymmetry oscillation of the two channels depends on the wavelength as well. Coincidentally, at $2.1 \mu \mathrm{m}$ the asymmetries of the two channels exhibit an opposite sign, reproducing the experimental observation [see Fig. 3(a)].

Comparing the current results to earlier work using nearinfrared few-cycle laser pulses [6,7], it is evident that the CEP control of electron localization in the dissociation of $\mathrm{D}_{2}$ is much more efficient for the BS channel when midinfrared pulses are applied. The larger wavelength implies a better match between the duration of the laser pulse and the dissociation time of the molecule, while the number of optical cycles is kept small. Thus for the few-cycle pulses used here, the wave packet in the $X$ state can be efficiently coupled to the $A$ state at large internuclear distances. Both aspects lead to the observed high degree of control of charge-directed reactivity in the dissociation reaction.

The simultaneous observation and control of the asymmetry resulting from the $\mathrm{BS}$ and the RCE dissociation channels is novel to the present study. Our time-dependent Schrödinger equation calculations identified the underlying mechanism leading to the observed asymmetries and reproduced well the general features of both channels that are controllable by the CEP. The details in the energy and CEP-dependent asymmetry provide strong constraints to theoretical models.

We acknowledge discussions of these results with B. Esry, I. Ben-Itzhak, and F. Martin. We are grateful for 
support by the Chemical Sciences, Geosciences, and Biosciences Division, Office of Basic Energy Sciences, Office of Science, U.S. Department of Energy, the National Science Foundation under CHE-0822646, the European network ATTOFEL, Laserlab Europe, the German Science Foundation via the Emmy-Noether program, and the Cluster of Excellence: Munich Center for Advanced Photonics.

[1] T. Brixner and G. Gerber, Chem. Phys. Chem. 4, 418 (2003).

[2] M. F. Kling and M. J. J. Vrakking, Annu. Rev. Phys. Chem. 59, 463 (2008).

[3] D. Ray et al., Phys. Rev. Lett. 103, 223201 (2009).

[4] M. Abel et al., Laser Photon. Rev. 5, 352 (2011).

[5] R. Weinkauf et al., J. Phys. Chem. 99, 11255 (1995).

[6] M. F. Kling et al., Science 312, 246 (2006).

[7] M. Kremer et al., Phys. Rev. Lett. 103, 213003 (2009).

[8] I. Znakovskaya et al., Phys. Rev. Lett. 103, 103002 (2009).

[9] B. Fischer et al., Phys. Rev. Lett. 105, 223001 (2010).

[10] I. Znakovskaya et al., Phys. Chem. Chem. Phys. 13, 8653 (2011).

[11] Y. Liu et al., Phys. Rev. Lett. 106, 073004 (2011).

[12] P.H. Bucksbaum et al., Phys. Rev. Lett. 64, 1883 (1990).
[13] T. Ergler et al., Phys. Rev. Lett. 97, 193001 (2006).

[14] F. He, A. Becker, and U. Thumm, Phys. Rev. Lett. 101, 213002 (2008).

[15] M. F. Kling et al., Mol. Phys. 106, 455 (2008).

[16] V. Roudnev and B. D. Esry, Phys. Rev. Lett. 99, 220406 (2007).

[17] D. Geppert, P. von den Hoff, and R. de Vivie-Riedle, J. Phys. B 41, 074006 (2008).

[18] P. von den Hoff et al., IEEE J. Sel. Top. Quantum Electron. (in press).

[19] X. Gu et al., Opt. Express 17, 62 (2009).

[20] B. Bergues et al., New J. Phys. 13, 063010 (2011).

[21] O. Ghafur et al., Rev. Sci. Instrum. 80, 033110 (2009).

[22] See Supplemental Material at http://link.aps.org/ supplemental/10.1103/PhysRevLett.108.063002 for a description of the theoretical methods and further calculations.

[23] H.-J. Werner and P. J. Knowles et al., MOLPRO, version 2006.1, 2006.

[24] J. L. Whitten, J. Chem. Phys. 39, 349 (1963).

[25] S. Huzinaga, J. Chem. Phys. 42, 1293 (1965).

[26] J. Hu et al., Phys. Rev. A 74, 063417 (2006).

[27] T. Nubbemeyer et al., Phys. Rev. Lett. 101, 233001 (2008).

[28] G. Sansone et al., Nature (London) 465, 763 (2010).

[29] F. Kelkensberg et al., Phys. Chem. Chem. Phys. 13, 8647 (2011).

[30] S. Gräfe and M. Y. Ivanov, Phys. Rev. Lett. 99, 163603 (2007). 